\title{
La crítica de la psicopatología sólo tiene sentido desde la psicopatología crítica
}

RESUMEN: En respuesta a un artículo anterior se argumenta a favor de una psicopatología crítica que tenga en cuenta los factores socioculturales que intervienen en los procesos de formación, consolidación e inteleccióón del síntoma psiquiátrico, en detrimento de una crítica de la psicopatología como empresa científica que asume una postura nominalista extrema, un concepto de trastorno mental exclusivamente evaluativo $\mathrm{y}$, por tanto, reduccionista sociológico, soslaya el debate actual acerca del propio concepto de trastorno mental y adopta una postura cercana a la denominada pospsiquiatría. PALABRAS CLAVES: psicopatología, trastorno mental, pospsiquiatría.

\begin{abstract}
In this reply to a former article it is argued for a critical psychopathology which accounts for the sociocultural factors that intervene in the development, consolidation and understanding of mental symptom, and against a criticism of psychopathology as a scientific project that assumes a strong nominalist view, adopts an evaluative reductionist concept of mental disorder, ignores the present debate on the concept of disorder and resembles the so-called postpsychiatry.
\end{abstract}

KEY WORDS: psychopathology, mental disorder, postpsychiatry

"Cuando quiero entender de entrada a alguien que me presentan sin mayores referencias, me las arreglo para sacar a Dali de algún cajón del diálogo. Si me dicen (sintetizo una opinión que puede durar diez minutos): "es un estupendo hijo de mala madre», siento que hay contacto, y que todo puede andar bien. Si, en cambio, la respuesta se corta por el lado de: "dejando aparte su pintura, es un ser moralmente despreciable», cierro el cajón y me despido lo antes posible, porque está claro que me ha tocado aguantar a un señor bien y pocas cosas me cuestan más que eso en la vida”.

Esta cita del añorado Julio Cortázar me viene siempre a la mente cada vez que inicio un intercambio de ideas con algún colega para mí desconocido, solo que, en vez de Dalí, yo suelo utilizar como test improvisado la opinión que a mi interlocutor le suscita la psicopatología actual. Como consecuencia de estos contactos profesionales uno ya ha elaborado una pequeña casuística de opiniones que reflejan las diferentes posturas que ante la psicopatología pueden adoptarse. 
En primer lugar estarían aquellos que piensan que la psicopatología que tenemos y que hemos heredado con pocos cambios de los alienistas del XIX es insuficiente y hay que cambiarla, bien porque no incluye aspectos relativos a la subjetividad del paciente (y ahí estarían desde las corrientes fenomenológicas a la psicoanalíticas), bien porque los elementos descriptivos son excesivamente subjetivos, difícilmente objetivables al proceder de la psicología popular (folk psychology) y, por tanto, poco aptos para la investigación científica ( $\mathrm{y}$ ahí se situarían desde las posiciones funcionalistas a ultranza de muchos modelos cognitivos y/o conductuales a los más extremos modelos eliminativistas que postulan sustituir el lenguaje psicopatológico que poseemos por otro neurobiológico más adecuado a las técnicas de imagen cerebral).

Por otro lado, un número considerable de profesionales sostiene que la psicopatología está bien como está y que con los 15-20 síntomas que manejamos (residuo del paso del tiempo sobre los 70-80 descritos a lo largo de la historia) nos es suficiente tanto para la práctica como para la investigación. Este segundo grupo tampoco es homogéneo; así, encontramos aquellos que, desde una posición esencialista, conciben a los síntomas expresados por el paciente como aspectos del fenómeno que debemos estudiar y, por tanto, lo único que hay que hacer es correlacionarlos (a ser posible en su presentación conjunta) con variables neurobiológicas duras; al mismo tiempo, hay quien defiende, desde una posición nominalista más o menos explícita, que síntomas y trastornos son constructos culturalmente determinados y que no obedecen a ningún aspecto de la realidad por lo que es igual que sean estos u otros: lo importante es que nos sirvan para otro fin mucho más importante (ayudar al paciente, planificar una técnica de intervención) (1).

Una primera lectura del breve pero interesante artículo del Dr. Fernández Liria publicado en un número anterior de esta revista (2) podría hacernos pensar que en él se defiende la segunda posición (la psicopatología está bien como está) pero en su versión nominalista extrema. Por decirlo de una forma coloquial y, pido disculpas de antemano, simplificadora: "es tontería que breguéis con esta historia de cambiar la psicopatología que tenemos pues no es más que un producto de una determinada ideología (en el sentido de conjunto de valores y visión de nuestra profesión de un determinado contexto sociocultural) y vuestro afán deriva de una sentimiento (inconsciente) de culpa ante esta situación y no de todo ese discurso de buscar la cientificidad y demás zarandajas".

Sin embargo, precisamente por su planteamiento, por las ideas que aduce en una bien armada argumentación y las conclusiones a las que parece llegar a partir de ésta (además de por la trayectoria intelectual de su autor) es por lo que a mi juicio el artículo merece una análisis exhaustivo que pudiera permitir un debate más profundo. Es lo que a continuación pretendo realizar.

\section{Principales tesis del artículo mencionado.}

Las principales afirmaciones recogidas en el citado artículo pueden resumirse en:

I. Pretender que la psicopatología sea la ciencia básica

a) de la teoría (a lo que llamaremos postulado A1) 


\author{
b) de la práctica clínica (postulado A2) \\ es "un espejismo"
}

II. Los postulados A1 y A2 asumen que la (historia de la) psicopatología

a) obedece exclusivamente a leyes internas (postulado B1)

b) determina la historia de la atención psiquiátrica (postulado B2)

III. La postura defendida en II es falsa ya que ocurre justamente lo contrario: es la historia de la atencióón psiquiátrica la que determina la de la psicopatología.

IV. Los determinantes de la atención psiquiátrica (y, por ende, de la psicopatología) son exclusivamente socioculturales

V. El espejismo queda explicado por no ver III ni IV y defender los postulados $\mathrm{A} 1, \mathrm{~A} 2, \mathrm{~B} 1, \mathrm{y} \mathrm{B} 2$.

El Dr. Fernández Liria denomina al punto de vista que defiende los postulados señalados Psicopatología crítica y al que contrapone las tesis I a V, Crítica de la psicopatología. Pues bien, en lo que sigue argumentaré que esta última no tiene sentido más que como parte integrante de la primera y que la posición del Dr. Fernández Liria, al menos en lo que puedo deducir de su artículo, defiende una postura nominalista o constructivista fuerte de la psicopatología, una concepción del trastorno mental como producto exclusivamente de factores socioculturales (en un sentido parecido al de Sedgwick) y por tanto, reduccionista sociológica, soslaya el debate actual entre posiciones esencialistas y prototípicas en cuanto a la conceptualización de los trastornos y, finalmente, adoptaría una visión de la psiquiatría de tintes posmodernos (eso que Bracken y Thomas denominan pospsiquiatría) (3).

\title{
2. Una concepción evaluativa del trastorno mental.
}

"En el síntoma no hay nada de sustancial (...) No hay en la naturaleza síntomas o enfermedades esperando a ser descubiertas por psicopatólogos (como no las hay esperando la mirada de anatomopatólogos, microbiólogos u otras) '”.

El Dr. Fernández Liria se sitúa claramente en una posición nominalista en cuanto a la existencia de síntomas y enfermedades. No son entes de la realidad, al modo de un vaso, una silla o un lago sino constructos culturales que no obedecen a nada de la realidad más que a las condiciones contextuales en que surgen. Por ello señala los elementos que a su juicio integran estos constructos: una cultura que define qué es enfermedad y qué no lo es, unos agentes encargados de tratarla y unas personas a las que se designa como pacientes. El propio autor señala, aunque tímidamente, que puede haber más elementos en esta ecuación pero, al no desarrollar cuales pueden ser estos y basar su argumentación en los tres señalados debo pensar que a su juicio éstos son los esenciales. Sin embargo esto no parece ser así, al menos en muchos de los síntomas de la psicopatología actual y en un gran número de los trastornos que tratamos los psiquiatras. Con ello no quiero decir que los tres elementos señalados no intervengan en toda conceptualización del síntoma y trastorno psicopatológico sino que no son exclusivos ni muchas veces los 
más importantes (al menos en lo que concierne a la intelección de los mismos). Y precisamente la psicopatología es la encargada de justificar cuando son estos u otros los factores esenciales. Por ejemplo, pensar que en la alucinación auditiva de uno de nuestros esquizofrénicos o de una paciente afecto de alucinosis alcohólica no existe algún elemento esencial distinto de los determinantes culturales que conforman los roles de paciente, médico/sanador y de entidad morbosa etiquetable es obviar la posible (y cada vez más clara) existencia de un mecanismo mental que puede estar alterado (por la causa que sea) y que da consistencia y estabilidad temporal a la existencia del síntoma. Nótese que no estoy diciendo que el propio concepto de alucinación, o de esquizofrenia, o de alucinosis, no sean constructos elaborados a partir de determinados contexto socioculturales sino que estas concepciones (por muy falibles, borrosas, cuestionables y modificables que sean) se generan, generalmente, alrededor de algún elemento esencial de la realidad que las hace consistentes. En este sentido los síntomas son datos que se refieren a determinados fenómenos psicopatológicos (4,5). Las teorías psicopatológicas utilizan los datos pero intentan explicar los fenómenos que subyacen. Los datos pueden modificarse, depender del contexto sociocultural o de observación pero una visión realista de la ciencia nos llevar a admitir que existen ahí fuera fenómenos (que desconocemos y que son nuestro objeto de estudio) que explican por qué un sujeto oye voces dentro de su cabeza en Cádiz, Tanzania y Finlandia de una forma tan aburridamente similar. Y lo mismo puede decirse de otros síntomas de otras especialidad (qué ocurre con el dolor, la fiebre, la hemorragia, la pérdida de visión, el temblor).

La concepción de que los trastornos mentales son exclusivamente juicios de valor no es nueva en psiquiatría. De hecho tiene un rancio abolengo, que entronca con posiciones antipsiquiáátricas como la de Szasz (6). Sin embargo, a diferencia de este autor, para quien la noción de trastorno físico sí está legitimada en cuanto tiene a la lesión como base objetivable (pero no la noción de trastorno mental que es una simple extensión de la anterior sin base objetivable y por tanto, una simple etiqueta, un mito que justifica el uso del poder médico), autores como Sedgwick que defienden la noción exclusivamente evaluativa sostienen que tanto la noción de trastorno físico como la de trastorno mental son juicios emitidos por un grupo social para expresar lo que es deseable o no según las normas e ideales sociales imperantes (7). Esta parece ser la posición defendida por el Dr. Fernández Liria al negar la existencia de la más mínima sustancialidad a los síntomas y trastornos mentales y fí́sicos. Nótese que Fernández Liria no dice que los juicios de valor son parte del concepto de trastorno o de síntoma (con lo que yo estaría totalmente de acuerdo) sino que éstos estáán formados únicamente por juicios de valor. Esta posición tiene, a mi juicio, importantes debilidades teóricas. En primer lugar no explica por qué situaciones indeseables para un grupo social (como la pobreza, la ignorancia, la mala suerte, el poco éxito con el sexo contrario o el dolor de la dentición) no son consideradas trastornos. Por otro lado, esta posición puramente evaluativa no explica por qué gente que comparte el mismo grupo sociocultural puede diferir en la consideración de una condición como trastorno a pesar de que pueda juzgarla como indeseable. Por ejemplo, todos los 
disidentes soviéticos que, por este motivo, eran internados en Hospitales Psiquiátricos no padecían de un trastorno mental a pesar de que todos contravenían el orden social imperante de la misma forma que todos los esclavos que huían de las plantaciones sureñas no estaban afectos de psicopatología (de la denominada drapetomanía) a pesar de que todos violaban el orden social en busca de la libertad (8). Precisamente el sustentar la consideración de síntoma y trastorno sobre bases exclusivamente evaluativas hace que no tengamos argumentos distintos a los evaluativos para discutir los ejemplos antes mencionados. Parafraseando al Dr. Fernández Liria, únicamente una crítica del sistema comunista soviético o del sistema de esclavitud americano podría ayudarnos a distinguir cuales de los internados tenía un trastorno o no. A mi juicio, esta crítica, aunque necesaria, tendría que ser completada por un modelo de síntoma y trastorno (esto es por una psicopatología) que, precisamente por asumir que hay mucho de evaluativo en juego en esos juicios, intentara anclarlos en bases científicas que nos sirvieran para explicarlos y distinguirlos del simple ejercicio de un sistema de valores, en este caso antihumanitario. Este anclaje tampoco está exento de riesgos (y posiblemente tampoco de valores), es falible y perfeccionable pero indudablemente de un mayor valor heurístico.

\section{El debate actual acerca del concepto de trastorno mental.}

El debate que introduce el Dr. Fernández Liria no es nuevo y, sin embargo, está de rabiosa actualidad en la literatura anglosajona, preferentemente la norteamericana (los británicos ya debatieron largo y tendido a lo largo de los 70 y 80). El mérito de hacer revivir este debate debe adjudicarse, a mi juicio, a Jerry Wakefield, un psicólogo con formación en trabajo social aplicado a Salud Mental y en Filosofía, quien en 1992, publicó un artículo titulado "El concepto de trastorno mental" y subtitulado "En la frontera entre los hechos biológicos y los valores sociales" (8). Para este autor, el concepto de trastorno (mental o físico) equivale al de disfunción dañina (harmful dysfunction), en donde disfunción es un fallo de los mecanismos internos (mentales o físicos) de un organismo para realizar funciones que han sido el resultado de la selección natural. Este modelo contempla dos elementos fundamentales en la conceptualización de todo trastorno: por un lado un juicio científico de que existe un fallo en una función determinada, por otro un juicio de valor acerca del daño que dicho fallo supone para el individuo. Ambos son necesarios para que pueda hablarse de trastorno (por ejemplo, la dextrocardia puede ser considerada una condición en la que la función natural - producto evolutivo - del organismo se ha visto alterada pero que, sin embargo no produce daño alguno al individuo según los valores de nuestra cultura: no es, por tanto, un trastorno). Dos aspecto de este modelo me parecen especialmente relevantes: por un lado la construcción del criterio científico sobre bases evolutivas, algo a lo que no estamos acostumbrados en psicopatología y sobre lo que ha llamado la atención, entre nosotros, Julio Sanjuán (9); por otro lado, la necesidad de una conjunción fáctica-evaluativa para dar sentido al concepto. El requerimiento del primer criterio distingue el modelo de Wakefield de las concepciones antipsiquiátricas (6) o puramente evaluativas (7), pragmáticas (10) o estadísticas (11); el del segundo de la concepción puramente biológica de Scadding (12) o del primer Kendell (13). 
El modelo de Wakefield puede ser calificado de esencialista (aunque quizá no puramente esencialista ya que precisa del componente evaluativo - daño) en el sentido de que para este autor existiría un proceso subyacente explicativo (selección natural que da lugar a funciones que pueden alterarse por la causa que sea) que da unidad a la categoría o concepto de trastorno. Este modelo no está exento de objeciones (por ejemplo, muchas funciones se distribuyen en un continuum y no es fácil distinguir función de disfunción, no siempre es tan simple la relación entre una y otra, o no es posible distinguir claramente entre criterio evaluativo y fáctico) pero, al mismo tiempo, soporta bastante bien todos los ejemplos y contraejemplos que se han aportado $(8,14,15)$.

La visión alternativa al modelo esencialista de Wakefield es la defendida por Lilienfeld y Marino $(16,17)$ para quienes, en la línea de las nuevas corrientes filosóficas de la elaboración de conceptos $(18,19)$ y a partir de las teorías de Wittgestein y Rosch, la conceptualización de una condición como trastorno (mental y físico) se realiza en virtud de la semejanza global de ésta con un prototipo más o menos ideal, por lo que no es posible definirlo basándose en rasgos o criterios categoriales. Desde esta perspectiva, un concepto así formado no posee un criterio o conjunto de criterios que le sirvan de anclaje científico y, por tanto, la cuestión de si ciertas condiciones son trastornos mientras que otras no lo son no puede ser respondida científicamente ya que no existen límites claros entre los trastornos y los no trastornos (aunque sí podría ser motivo de investigación científica el por qué ciertas condiciones tienden a ser consideradas trastornos en un momento determinado). Por ejemplo, el concepto de lago no se corresponde con un punto genuino de demarcación en el mundo real por lo que la distinción entre lago, laguna, charca, embalse, o incluso mar, no está sujeta al escrutinio científico: esta distinción se basa, en parte, en el tamaño de la masa de agua, en parte en convenciones sociales (si puede o no albergar un barco, si está o no destinado a un fin, etc).

Lilienfeld y Marino tienen especial cuidado, sin embargo, en dejar claro que con la consideración de trastorno como un concepto prototípico o roschiano no quieren decir que éste sea un constructo mental que no se corresponde con nada de la realidad. Por el contrario, este tipo de conceptos surge de múltiples experiencias con entidades reales cuyos rasgos más consistentes van a ayudar a conformar el prototipo. Así, el concepto de lago surge de la observación de una masa de agua real, independientemente que otras consideraciones no fácticas sean relevantes para distinguirlo de otros conceptos limítrofes y lo mismo podría decirse del concepto de trastorno mental. Para estos autores, por ejemplo, la disfunción evolutiva apuntada por Wakefield puede ser una de las características del prototipo pero no la única ni la esencial. Otras características como la inadaptación a un contexto, la percepción de que algo funciona mal o la creencia de que se necesita tratamiento, puede contribuir tanto o más a la conformación del prototipo y, por tanto, del concepto, como la disfunción de Wakefield.

Resulta curioso (y alentador) constatar en las más recientes aportaciones de sus principales promotores $(14,15,17)$ que las dos posturas en un principio antagónicas tienden a converger de una forma muy interesante y difieren no tanto en si asumen o no 
una postura esencialista sino en el tipo de esencialismo defendido y en la naturaleza de los aspectos esenciales: para unos la disfunción evolutiva (esencialismo de caja negra - estipula que debe haber una esencia real que desconocemos y que hay que estudiar) (15), para otros la idea psicosocialmente determinada de que algo va mal en el organismo (exista ese algo o no) es lo que determina la conformación del prototipo (esencialismo psicológico) (20). En resumidas cuentas, para unos el concepto de trastorno mental tiene un anclaje en la realidad aunque sea desconocido para nosotros, mientras que para los otros dicho concepto es un producto psicosocial de la experiencia repetida con las personas supuestamente afectas independientemente de que exista realmente alguna disfunción. Para los primeros el concepto de trastorno mental es un concepto científico, para los otros no. Sin embargo ninguna de las dos posturas niega la posibilidad de que existan disfunciones reales y que por tanto puedan ser estudiadas científicamente.

\section{Un cierto sabor a pospsiquiatría.}

En los últimos tiempos entre los teóricos de la salud en Gran Bretaña se han acuñado las expresiones medicina posmoderna (21) o pospsiquiatría (3) para referirse a una supuesta nueva dirección en la teoría y práctica médica o de la Salud Mental que, en sintonía con la filosofía posmoderna, propondría un rechazo de la creencia de que la ciencia y la tecnología puedan resolver los problemas del ser humano y de su grupo social, un cuestionamiento del control médico de las intervenciones coercitivas y de la importancia dada a las circunstancias y variables individuales en la comprensión de los trastornos mentales. Mientras rechazan la herencia de la Ilustración y la entronización de la razón y del individuo, estos autores proponen un mayor énfasis en el contexto sociocultural y político, en el papel de los grupos tanto en la génesis como en el manejo de los trastornos y en una orientación ética antes que científica de la práctica clínica. En consecuencia proponen superar el pensamiento moderno en psiquiatría para dar una mayor participación a los usuarios y redefinir las funciones de los profesionales de la Salud Mental. Aunque estos autores no incluyen recomendaciones específicas, intentan argumentar lo anterior sobre la base de a) una visión foucaultiana del origen de la psiquiatría; b) una crítica de la visión de la locura como algo interno al individuo, que los autores atribuyen a...jJaspers!; c) una visión de la propuesta moderna en psiquiatría equivalente a la psiquiatría biologicista (o reduccionista biológica) y al sistema DSM; y d) una advertencia sobre las limitaciones del discurso científico-tecnológico para ejercer adecuadamente el indudable poder coercitivo que la sociedad pone en manos de los psiquiatras (3).

La propuesta de superar la agenda de modernización de la psiquiatría podría ser atractiva...si la psiquiatría se hubiera modernizado. A mi juicio, no es así (al menos no del todo). Precisamente muchos de los problemas que soporta la psiquiatría como disciplina científica se deben a la reticencia con que se aplican los presupuestos filosóficos de la ciencia pospositivista y a la preferencia por la apariencia de rigor ante la rigurosidad $(22,23,5)$. Pensar que la modernidad psiquiátrica equivale a la creencia en el DSM y en la redención por la psiquiatría biologicista es, cuando menos, ingenuo. Por otra parte, 
el señalar el locus de control (24) interno como el lugar de búsqueda de los presuntos mecanismos alterados de la psicosis (propuesta que evidentemente es muy anterior a Jaspers) no deja de ser una hipótesis que ha dirigido la investigación sobre los trastornos mentales a lo largo de la historia y que, como todas las hipótesis, podría ser refutada cuando se demuestre que otros loci de control son heurísticamente más fructíferos pero no antes ni por decreto filosófico desde una posmodernidad de límites borrosos. Además, y por si fuera poco, tampoco creo que, como señalan Bracken y Thomas, la psiquiatría tecnocientífica y la concepción individual y liberal de la Ilustración hayan perjudicado al enfermo mental, independientemente de que, obviamente, no han resuelto todos sus problemas. Muchos de los derechos obtenidos y gran parte de la lucha por su autonomía (entre ellos el derecho a rehusar el tratamiento y a supervisión judicial ante un ingreso involuntario) son el producto precisamente de esa concepción ilustrada. Reforzar lo conseguido y luchar por mejorar los derechos aún conculcados del enfermo mental debe ser un reto para todos los psiquiatras independientemente de que asuman un paradigma psicopatológico determinado: el primer enunciado pertenece a un discurso ético, previo y preeminente ante toda actividad de conocimiento, mientras que el segundo es científico-tecnológico y no necesita renunciar a una concepción del individuo heredera de la Ilustración. Es probable que éste segundo no deba ser el único que justifique la detención del poder coercitivo depositado socialmente en la psiquiatría pero de ahí no se deduce que tengamos que renunciar a un discurso propio cuyo objetivo sea la intelección de la conducta psicopatológica. Sería como lo expresado por el dicho aquel de que al quitar el tapón del baño mientras bañamos al niño éste se va por el desagüe junto con el agua sucia.

Por otro lado, independientemente de lo brillante y sugerente que nos resulte la visión foucaultiana del devenir de las ideas psiquiátricas, lo cierto es que resulta en el mejor de los casos parcial y en el peor profundamente especulativa y tramposa. La psicopatología decimonónica puede que haya surgido en un contexto asistencial determinado (asilar) pero de ahí no se deduce que el devenir de las ideas psicopatológicas pueda reducirse a los distintos cambios de escenarios sociopolíticos $(25,26,27)$. Existe una historia de la psicopatología que, aunque obviamente entrelazada con la historia de las instituciones, mantiene unos hilos conductores independientes y que no pueden ser reducidos a ésta, como parece defender Fernández Liria.

Es evidente que el sueño de la razón puede producir monstruos pero esta afirmación puede tomarse en un doble sentido: por un lado el confiar únicamente en ella como garante de la solución de todos los problemas puede abocar irremediablemente al fracaso (o a la tiranía de lo médico); pero también confiar todas las naves a una alternativa de la racionalidad psicopatológica (por muy deficitaria que ésta sea) lleva al reinado de lo irracional y tampoco garantiza el éxito de la empresa. En este sentido, más que un pensamiento psiquiátrico débil necesitamos fortalecer el pensamiento psiquiátrico que tenemos y por tanto, más y mejor razón (28). 


\section{5. ¿Es la psicopatología el (único) fundamento de la práctica clínica?}

En la base de la argumentación del Dr. Fernández Liria existe una confusión, probablemente buscada, entre varios niveles discursivos en mi opinión diferentes: el de la investigación psicopatológica, el de la teoría acerca de la práctica asistencial y el propio de la práctica. Correspondería a lo que Pierre Bourdieu denominaba respectivamente niveles de la teoría, de la teoría de la práctica y de la práctica (29), o por decirlo de otro modo, científico, tecnológico y técnico (23). La psiquiatría se halla en una situación intersticial entre estos tres niveles y, por tanto, debe expresarse en todos ellos de forma específica. Eso no quiere decir que los niveles discursivos sean completamente independientes o impermeables pero tampoco que puedan reducirse a uno: la relación entre niveles no es de subordinación intrínseca sino contextual y dependiente de las necesidades y objetivos de la empresa en cuestión. Está claro que los factores determinantes del discurso tecnológico/técnico no tienen por qué ser exactamente los mismos que los del nivel científico: por ejemplo, una visión de la sanidad publica como algo cada vez más participativo para los usuarios no necesariamente es contradictorio con que la investigación neuropsicológica de las alteraciones de la memoria en ciertas esquizofrenias nos lleve a pensar que puede existir una anomalía en la recuperación de contenidos relativos a la memoria autonoética, relacionada con contenidos autobiográficos y el self (30). Lo importante es que un servicio sanitario organizado para dar respuesta a las exigencias de una filosofía asistencial determinada integre la información proveniente de la ciencia psiquiátrica para mejorar la atención dispensada. En este sentido es posible que pueda hablarse del desideratum de una confluencia de narrativas (31) pero no como un a priori desde el que el discurso de la teoría se ve sometido a una interpretación exclusivamente sociologista de su sentido.

El admitir que los niveles tecnológicos/técnicos dependen de factores de índole diferente a los que favorecen el desarrollo del nivel científico o de la investigación psicopatológica no quiere decir que no existan grandes áreas de solapamiento entre ellos. Esto evita la idea romántica de la psicopatología como una ciencia pura independiente de los avatares asistenciales, socio-políticos y culturales. Antes al contrario, el discurso científico está preñado de valores y disvalores que habrá que tamizar de forma crítica pero preferentemente desde la psicopatología sin que tengamos que prescindir del nivel de la teoría y situarnos en otros niveles. El nivel teórico no está exento de influencia sociales: es precisamente lo que una buena psicopatología crítica debe discernir (cuándo un trastorno aparece simplemente porque un determinado fármaco se ha lanzado al mercado y necesita una nueva indicación, cuándo existen criterios de delimitación del mismo, por quéé Kraepelin tuvo éxito en detrimento de Wernicke o Jaspers en detrimento de Chaslin, qué razones empujan al abandono del concepto de pseudoalucinación o por qué los análisis actuales del insight en la psicosis son tremendamente confusos). Nótese que no estoy renunciando al tipo de análisis que plantea Fernández Liria: simplemente lo integro en el ámbito que parece más pertinente. Asumo el adjetivo crítico pero para calificar a lo que me parece sustantivo: la psicopatología. Sustantivar la psicopatología no es convertirla en un icono ni admitir que dicte cómo se organiza el nivel asistencial: 
los factores que deben determinar esto último no son exclusiva ni fundamentalmente psicopatológicos aunque una buena organización asistencial que tenga en cuanta las características (y las causas) del sufrimiento de sus usuarios debe así mismo tener en cuenta lo aportado por una psicopatología elaborada como un lenguaje bien hecho, como una disciplina científica adaptada a los conocimientos actuales y no como una reliquia intocable del pasado o como un mero epifenómeno de instancias sociopolíticas por desenmascarar.

\section{Psicopatología crítica vs crítica de la psicopatología : conclusión}

En conclusión, lo que Fernández Liria denomina psicopatología crítica asume el postulado A1 de su argumentación (psicopatología como ciencia básica de la teoría) pero no necesariamente el A2 (ya que, aunque el discurso psicopatológico pueda ser importante, otras consideraciones o discursos son más determinantes en la teoría de la practica y en la practica). Por otra parte, no asume el postulado B1 (ya que no exclusivamente se debe a leyes internas aunque tampoco exclusivamente a leyes externas) ni el B2 (la psicopatología no determina - ni ahora ni nunca - la organización de la asistencia). Finalmente, rechaza, como hemos visto en las páginas anteriores, III, IV y, en consecuencia, $\mathrm{V}$ es un non sequitur. La crítica de la psicopatología tiene más sentido si se hace desde una psicopatología crítica.

* Agradecimientos: Agradezco al Profesor Rogelio Luque sus valiosas sugerencias.

\section{BIBLIOGRAFÍA.-}

1. Villagrán, J.M. ¿Necesitamos una psicopatología descriptiva? Archivos de Psiquiatría, 2001, 64 (2), 97-100.

2. Fernández Liria, A. De las psicopatologías críticas a la crítica de la psicopatología. Revista de la Asociación Española de Neuropsiquiatría, 2001, 21, 80, 57-69.

3. Bracken, P., Thomas, P. Postpsychiatry: a new direction for mental health. British Medical Journal, 2001, 322 (7288), 724-727.

4. Bogen, J., Woodward, J. Saving the phenomena. Philosophical Review, 1988, 97, 303-352.

5. Villagrán, J.M. Hacia una psiquiatría biológica no reduccionista: los modelos interdisciplinares. En VVAA Hechos y valores en psiquiatría. Madrid: Triacastela (en prensa).

6. Szasz, T. The myth of mental illness: foundations of a theory of personal conduct. Rev. ed. New York: Harper \& Row, 1974.

7. Sedgwick, P. Psycho politics. New York: Harper \& Row, 1982

8. Wakefield, J.C. The concept of mental disorder. American Psychologist, 1992, 47, 3, 373-388.

9. Sanjuán, J. (ed) Evolución cerebral y psicopatología. Madrid: Triacastela, 2000.

10. Taylor, F.K. The medical model of the disease concept. British Journal of Psychiatry, 1976,128, 588-594.

11. Cohen, H. The evolution of the concept of disease. En Caplan, A.L., Engelhardt, H.T., McCartney, J.J. (eds), Concepts of health and disease: interdisciplinary perspectives. Reading, MA: Addison-Wesley, 1981, pp. 209-220. 
12. Scadding, J.G. The semantic problem of psychiatry. Psychological Medicine, 1990, 20, 243-248.

13. Kendell, R.E. The concept of disease and its implication for psychiatry. British Journal of Psychiatry, 1975, 127, 305-315.

14. Wakefield, J.C. Evolutionary versus prototype analyses of the concept of disorder. Journal of Abnormal Psychology, 1999, 108, 374-399.

15. Wakefield, J.C. Mental disorder as a black box essentialist concept. Journal of Abnormal Psychology, 1999, 108, 465-472.

16. Lilienfeld, S.O., Marino, L. Mental disorder as a Roschian concept: a critique of Wakefield's "harmful dysfunction" analysis. Journal of Abnormal Psychology, 1995, 104, 411-420.

17. Lilienfeld, S.O., Marino, L. Essentialism revisited: evolutionary theory and the concept of mental disorder. Journal of Abnormal Psychology, 1999, 108, 400-411.

18. Peacocke, C. A study of concepts. Cambridge, MA: MIT Press, 1992.

19. Mechelen, I.V., Hampton, J., Michalski, R.S., Theuns, P. Categories and concepts. Theoretical views and inductive data analysis. London: Academic Press, 1993.

20. Medin, D., Ortony, A. Psychological essentialism. En Vosniadou, S., Ortony, A (eds), Similarity and analogical reasoning. Cambridge: Cambridge University Press, 1989, pp. 179-195.

21. Muir Gray, J.A. Postmodern medicine. Lancet, 1999, 354, 1550-1553.

22. Villagrán, J.M., Olivares, J.M. ¿Hay alguna psiquiatría que no sea biológica? Revista de la Asociación Española de Neuropsiquiatría, 1995, 15 (54), 528-542.

23. Villagrán, J.M., Luque, R. Bases epistemológicas de la teoría y práctica psiquiátricas. En Luque, R., Villagrán, J.M. (eds) Psicopatología descriptiva: nuevas tendencias. Madrid: Trotta, 2000, pp. 75-106.

24. Bechtel, W., Richardson, R.C. Discovering complexity: decomposition and localization as strategies in scientific research. Princeton, NJ: Princeton University Press, 1993.

25. Mora, G., Brand, J.L. (eds) Psychiatry and its history. Methodological problems in research. Springfield, Illinois: Charles Thomas, 1970.

26. Marx, O.M. What is the history of psychiatry II. History of Psychiatry, 1992, 3, 293-301.

27. Berrios, G.E. The history of mental symptoms. Cambridge: Cambridge University Press, 1996.

28. Villagrán, J.M. El surgimiento de la (neuro)ciencia cognitiva: ¿una nueva moda o, por fin, una propuesta unitaria para la ciencia psiquiátrica? Revista Gallega de Psiquiatría y Neurociencias, 2002, 5 (1), 1-2.

29. Bourdieu, P. Esquisse d'une théorie de la pratique. Genève: Droz, 1972.

30. Lorente, E., Villagrán, J.M. Autobiographical memory in schizophrenia: a conceptual review (Poster). III International Conference on Memory (ICOM-3). Valencia, Julio 16-20, 2001.

31. Fernández Liria, A. Una confluencia en las narrativas. En Fernández Liria, A., Rodríguez Vega, B. La práctica de la psicoterapia; construcción de narrativas terapéuticas. Bilbao: Desclée de Brouwer, 2001.

* Psiquiatra. Coordinador de la Unidad de Hospitalización Psiquiátrica. Hospital de Jerez (Cádiz). Servicio Andaluz de Salud.

e-mail: jvilla@maptel.es

Fecha de recepción: $12-\mathrm{V}-02$ 